\title{
CLASSIFICATION TECHNIQUE OF MACHINE LEARNING AS SPECIES DISTRIBUTION MODEL FOR EXOTIC FISH IN RIVERS
}

\author{
Risa SHIROYAMA ${ }^{1}$, Chihiro YOSHIMURA ${ }^{2}$ \\ ${ }^{1}$ Student Member of JSCE, Doctoral student, Dept. of Civil Engineering, Tokyo Institute of Technology \\ (2-12-1-M1-4, Ookayama, Meguro-ku, Tokyo 152-8552, Japan) \\ ${ }^{2}$ Member of JSCE, Dr. of Eng., Assoc. Professor, Dept. of Civil Engineering, Tokyo Institute of Technology \\ (2-12-1-M1-4, Ookayama, Meguro-ku, Tokyo 152-8552, Japan)
}

\begin{abstract}
The prevalence of exotic species has been a major ecological problem all over the world, and Japan is no exception. This study modelled distributions of five exotic fish species (channel catfish, bluegill, largemouth bass, smallmouth bass and mosquitofish) in major rivers in Kanto region by using classification techniques: classification and regression trees (CART) and random forest (RF). National Census on River Environments was used as a response and predictor variables. Both models showed high prediction accuracy for all exotic species, and RF outperformed CART. Suitable habitat ranges of the fish estimated by RF were well accorded with the ranges reported based on observations. Additionally, the result was presented also as the species distribution map as an example of its application. Overall, this research demonstrated the importance of the combination between advanced statistical approach (CART and RF) and detailed environmental data.
\end{abstract}

Key Words : Classification and regression trees, Random forest, Exotic fish species, Kanto region, NCRE

\section{INTRODUCTION}

There are increasing needs of understanding species distribution for environmental conservation purposes. To understand the species distribution, species distribution models (SDM) have been developed to understand and predict plant or animal species distribution ${ }^{1)}$. They are implemented also to river ecosystem over the past decade ${ }^{2}$. Generalized linear models (GLM) and generalized additive models (GAM) are widely known for SDMs. However, these conventional statistical approaches have been criticized for employing unrealistic assumptions. For example, both GLM and GAM assume observations are independent; not auto-correlated over time and space. Ecological data are generally complex enough to contain a non-linear relationship between response and predictor variables and collinearity within predictor variables. In recent years, by grace of advances computer technology, more sophisticated statistical approach such as classification and regression trees (CART) and random forest (RF) has been established. Their capability for modelling the presence and absence of freshwater fish have been supported by previous studies ${ }^{2), 3)}$, however, such approaches are rarely applied to the Japanese freshwater fish species.

In order to understand river ecosystem, National Censuses on River Environments (NCRE) has been conducted by the Ministry of Land, Infrastructure and Transport (MLIT) of Japan since $1990^{4}$. This survey aims to collect riverine species information to apply river managements and promote studies on riverine ecosystem ${ }^{5}$. Despite the large accumulation of useful data, unfortunately they are not effectively used. For example, few researchers tackled with this datasets for finding new ecological insights related to the river conservation and management ${ }^{6}$. Thus, this dormant environmental data are expected to be utilized possibly with the help of advanced information technology.

The prevalence of the invasive exotic species has been a major ecological problem worldwide. They are notoriously known as one of the factors of diversity loss through predation, parasitism and cross-fertile for indigenous species ${ }^{7)}$. They are mainly introduced by human activity and spread 
beyond their native range ${ }^{8)}$. Colonization speed of these species has been increased along with rapid globalization. In Japan, these invasive exotic species has been caused harmful effects on existing species. Ministry of Environment constructed a detailed list of endangered species, and approximately 167 of $400(42 \%)$ freshwater fish species are listed as threatened species in $2013^{9)}$. Thus, describing the distribution of invasive exotic species is imperative for protecting state of nature.

The objective of this paper is to develop SDMs of exotic freshwater fish species using both newly established statistical techniques and NCRE survey data. CART and RF were used for model construction to investigate whether these models are applicable to the fish species distribution. The availability of NCRE survey data was also examined. Additionally, the consistency in suitable habitat ranges from both modelled result and reported habitat information were investigated.

\section{STUDY AREA AND DATASETS}

\section{(1) Study area}

In this study, seven rivers in Kanto region ${ }^{10)}$ were targeted for model construction (Table 1). This region has a large plane in central part and mountain area in North-West and open to the Pacific Ocean in East part. Additionally, this area plays a key role in political and economic center of Japan. In each river, sample areas were set up (131 in total). Each sample area has one or more sample points. In this study, the following analysis was made based on the information from each sample point. The number of all sample points was 527 . After removing invalid sample points which have missing values, 425 samples were used in the analysis.

\section{(2) Target fish and habitat conditions}

In Japan, "exotic species" has defined those introduced after Japan abandons its national isolation policy ${ }^{11)}$. Ministry of Environment defined the list of regulated living organisms under the

Table 1 River description in Kanto region

\begin{tabular}{|c|r|r|r|r|r|}
\hline River & $\begin{array}{c}\text { Area } \\
\left(\mathrm{km}^{2}\right)\end{array}$ & $\begin{array}{c}\text { Average } \\
\text { altitude }(\mathrm{m})\end{array}$ & $\begin{array}{c}\text { No of } \\
\text { area }\end{array}$ & $\begin{array}{c}\text { No of } \\
\text { points }\end{array}$ & $\begin{array}{c}\text { Year of } \\
\text { sampling }\end{array}$ \\
\hline \hline Ara & 2,920 & 344 & 17 & 41 & 2007 \\
\hline Kuji & 1,490 & 324 & 11 & 23 & 2010 \\
\hline Naka & 3,270 & 336 & 11 & 21 & 2010 \\
\hline Sagami & 1,560 & 693 & 3 & 9 & 2007 \\
\hline Tama & 1,240 & 553 & 16 & 55 & 2006 \\
\hline Tone & 16,480 & 469 & 58 & 208 & 2009 \\
\hline Tsurumi & 240 & 46 & 15 & 68 & 2009 \\
\hline
\end{tabular}

Invasive Alien Species Act (IAS). It lists the organisms which potentially affect native species through predation, and give harmful effect on human and agriculture. Growing, selling and importing them are prohibited in law since June $2005^{12)}$.

This study modelled five exotic fish species to cover various prevalence range, channel catfish (Lctalurus punctatus), bluegill (Lepomis macrochirus), largemouth bass (Micropterus salmoides), smallmouth bass (Micropterus dolomieu) and mosquitofish (Gambusia affinis). Their prevalence ranged from $5.6 \%$ to $26.3 \%$ of all sample points; channel catfish (10.8\%), bluegill (26.3\%), largemouth bass (13.9\%), smallmouth bass (6.1\%) and mosquitofish (5.6\%). All of them are listed on IAS and introduced 19th century for fishery, edible purpose and the pest control. Species richness and population density of native species have been decreased after their colonization ${ }^{7)}$.

All the data used in this analysis were gathered by NCRE during 2006-2010. They targeted to conduct survey for Class A River in Japan. In each river, several sampling points were set and species presence/absence information and environmental data were collected at the points ${ }^{13}$. This paper investigated five exotic fish species and implement statistical analysis to predict their presence/absence using eight environmental variables; distance from river mouth, longitudinal slope, spring and autumn depth, spring and autumn velocity and spring and autumn temperature at each monitored site. In this study, spring covers from December to May, and autumn covers from June to November. Target fish were counted as "presence" if they were observed either spring or autumn.

\section{METHODS}

\section{(1) Classification and regression tree (CART)}

CART is a non-parametric technique which extracts a non-linear relationship between species response and environmental variables. The aim of the tree based method is to classify existing and new observation into the classes using the numeral and /or categorical explanatory variables ${ }^{14)}$. This model partition the space into two subsets to increase the purity of the spaces. The same procedure, space dividing, continue until the further partition no longer increase purity ${ }^{15}$. This model provides more interpretable results and can deal with missing values in both response and explanatory variables ${ }^{16)}$.

Classification tree has become increasing important because of its conceptual simplicity and computational efficiency, and applied not only ecological problems but also diverse problems such 
as remote sensing, character recognition and medical diagnostics ${ }^{17}$.

When the predicted outcome can be considered a real number (e.g. the price of a house, or a patient's length of stay in a hospital), this method is called regression trees and if outcome are class (e.g. presence/absence) called classification trees. Both tree method are known collectively as classification and regression trees (CART) ${ }^{18)}$.

\section{(2) Random forest (RF)}

Random forest was introduced by Breiman $(2001)^{19)}$. This model start building a large number of trees (make "forests") from bootstrap samples derived by original dataset. In this paper, 1000 trees are made for prediction ${ }^{20)}$. After finishing the tree growing, the result is averaged. The unique process of random forests is that each tree grown with a randomized subset of explanatory variables and this process improve their prediction accuracy ${ }^{21)}$.

Conventional statistical approaches indirectly measure a variable importance using Akaike's Information Criterion (AIC) ${ }^{22)}$. In RF the process is totally different. The value of the targeted variable is randomly permuted before a misclassification rate (or node impurity) are calculated. The difference between original and modified misclassification rate (or node impurity) is the measure of the variable importance $^{14)}$. This process is conducted for all variables, and they are listed on the variables importance table. The advantages of the random forests are robust to outliers and noise ${ }^{19)}$, and high prediction accuracy.

\section{(3) Evaluation of model performance}

Samples (425) were divided into two datasets randomly: training data (218) and test data (207). Training data are used for model construction and test data are used for model validation (Figure 1). For modelling, we used CART and RF package on R 3.0.1(R Core Team 2014).

Model performance was evaluated using the receiver operating curve (ROC). A ROC plot is obtained specificity for $\mathrm{X}$-axis and sensitivity for $\mathrm{y}$-axis for all available thresholds ${ }^{23)}$; both specificity and sensitivity are calculated using confusion matrix

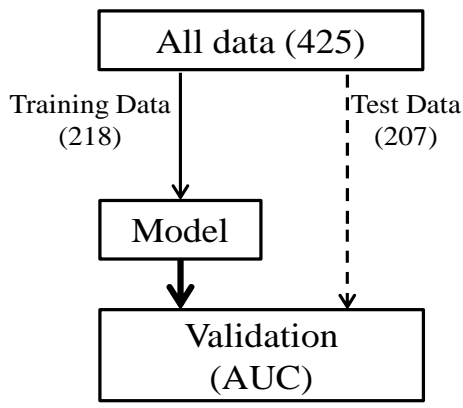

Figure 1 Flow of model development
(Table 2). This matrix is composed from observation (presence, absence) and prediction from the model (presence, absence). Each four cells are classified as True Positive (TP), False Negative (FN), False Positive (FP) and True Negative (TN). Using these four categories, specificity and sensitivity are calculated for all possible threshold value in order to draw ROC curve.

The area under the ROC curve is called AUC and this value indicates the predictive ability of presence/absence of the species. For instance, a score $50 \%$ indicates that the model does not have the discriminant ability (same as a coin toss), while a score $100 \%$ indicate that the presence and absence are perfectly discriminated.

In addition to predicting accuracy measure, a ROC analysis provides binary output of presence/absence. The output of the statistical model usually provide probability of the species presence/absence, however, the information presented by binary output, such as presence or absence, are more practical than presented by probability ${ }^{24)}$. The plotted ROC points which close to the upper-left corner is selected as optimal point and a threshold probability is calculated.

\section{RESULTS AND DISCUSSION}

\section{(1) Predicting performance}

ROC curve and AUC values were calculated for five exotic species using CART and RF. These output suggested that both CART and RF have high prediction accuracy for training data. The average AUC were $83.7 \%$ and $99.7 \%$ for CART and RF respectively (Figure 2, Table 3). Additionally, RF has good predicting accuracy for test data $(>80 \%$ in average). In the previous study, model AUC was calculated for 26 endangered fish species in Japan, and average AUC is $91.5 \%$ for training data ${ }^{25)}$. They used generalized linear model (GLM) for statistical modelling. These results suggest that RF showed superior performance than CART and previous research (GLM). RF is classified as ensemble method. This method generates the set of classifiers and their individual decisions are combined in some way (typically voting or weighted voting). RF

Table 2 Confusion matrix

\begin{tabular}{|c|c|c|c|}
\cline { 3 - 4 } \multicolumn{2}{c|}{} & \multicolumn{2}{c|}{ Observed } \\
\cline { 3 - 4 } Predicted & Presence & Absence \\
\cline { 3 - 4 } & Presence & $\begin{array}{c}\text { True Positive } \\
(\mathrm{TP})\end{array}$ & $\begin{array}{c}\text { False Positive } \\
(\mathrm{FP})\end{array}$ \\
\cline { 2 - 4 } & Absence & $\begin{array}{c}\text { False Negative } \\
(\mathrm{FN})\end{array}$ & $\begin{array}{c}\text { True Negative } \\
(\mathrm{TN})\end{array}$ \\
\hline \multicolumn{3}{|c|}{$\begin{array}{c}\text { Specificity=TN/(TN+FP) } \\
\text { Sensitivity }=\mathrm{TP} /(\mathrm{TP}+\mathrm{FN})\end{array}$}
\end{tabular}


combines the generated multiple tree result to construct a model. Thus, the outcome of this approach is often more accurate than any of its individual classifiers that make them up ${ }^{26)}$.

Moreover, these results also support the idea that this NRCE data are useful for predicting species distribution. The fish habitat information seems to be well captured by these predictor variables. In order to improve the prediction accuracy, additional explanatory variables are needed to be incorporated. In this study, only eight explanatory variables were used. However, fish colonization might be affected also by other variables such as longitude, latitude and dissolved oxygen. Moreover, only spring and autumn segments are used for seasonal information. However, some fish move to different place seasonally. To incorporate this information, time scale variables (i.e. year, month) could be a reasonable indicator for seasonality.

Additionally, bootstrapped method is an effective way to support this result. In this study, AUC was calculated for only one time for one set of training and test data. This means that if we use a different set of training (or test) samples, the result might be changed. It is said that species prevalence affects the prediction accuracy ${ }^{24}$, however, the result shows there is no significant relationship between the species prevalence and prediction accuracy ( $p$-value $=0.398$ ). Thus, AUC needs to be recursively calculated to investigate the relation between the species prevalence and prediction accuracy. Moreover, AUC was used for validation metric. To make a robust conclusion, other validation metric (i.e. Cohen's Kappa) need to be calculated.

\section{(2) Variable contribution}

Table 4 is a summary of the variable importance calculated by RF. The values are the percent increase in mean squared error when targeted variable is randomly permuted. A large value suggests its importance (thus error rate was increase by permutation). This information is useful to find the factor which affects the colonization of target species.

Here, we take bluegill as an example to discuss the consistency in suitable habitat range for both modelled result and habitat information collected by survey. The model result suggests that the probability of existence becomes increasing where near the middle and lower parts of the river (Figure 3a). The survey result also shows that this species exists where the slope is gentle. In the Figure $\mathbf{3 b}$ and Figure 3c, model result show that the magnitude of presence is high where the velocity is less than $50 \mathrm{~cm} / \mathrm{s}$. For both figures, the survey results also show high fish existence in slow velocity range. These results support the idea that the model results are well accorded with the real survey data. Additionally, previous literature reported that this fish prefers the gentle stream and intricate condition of under water, such as shore protection and water weed $^{27228)}$. This information is also corresponded with the model results.

\section{(3) Application for practical use}

Projection map was made to seek the applicability of this research into the practical use. The fish distribution model was made using training data and $\mathrm{RF}$, and then projection was conducted for test data. Figure 4 is the projection map of Naka river for bluegill drawn by google map. Each sample area has one or more sample points, and model construction was made based on sample points. However, the points in same sample area have same location information. Thus, the result calculated in each points are combined into one sample area, and the seven arrows shown in the map is the result based on sample area.

Observation and model prediction are
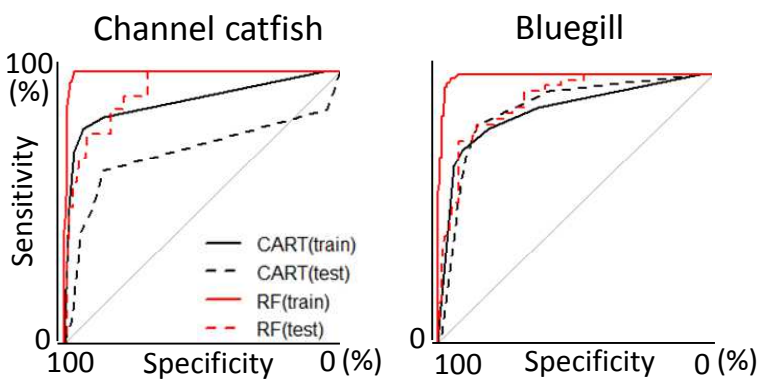

Largemouth bass

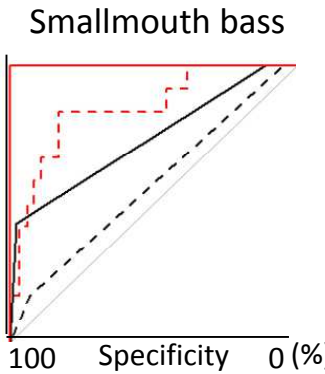

Mosquitofish
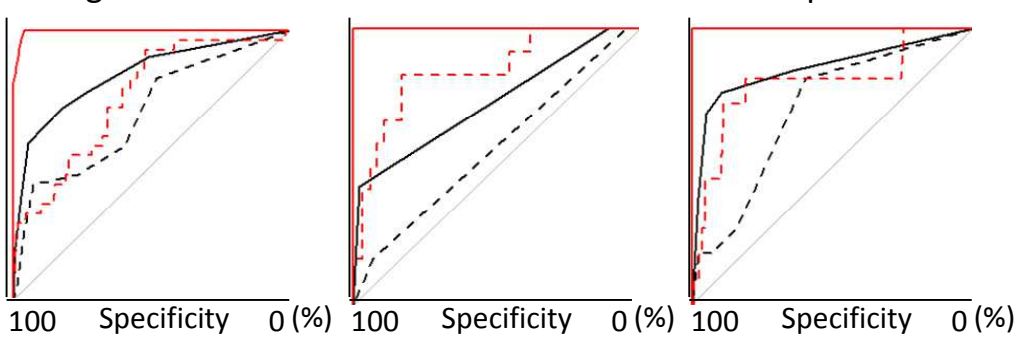

Figure 2 ROC curve

Table 3 AUC value for five fish species

\begin{tabular}{|c|c|c|c|c|c|c|c|c|c|c|c|c|}
\hline & \multicolumn{2}{|c|}{ Channel catfish } & \multicolumn{2}{|c|}{ Bluegill } & \multicolumn{2}{|c|}{ Largemouth bass } & \multicolumn{2}{|c|}{ Smallmouth bass } & \multicolumn{2}{|c|}{ Mosquitofish } & \multicolumn{2}{|c|}{ Average } \\
\hline & Train & Test & Train & Test & Train & Test & Train & Test & Train & Test & Train & Test \\
\hline CART & 89.2 & 70.4 & 86.3 & 87.2 & 83.8 & 69.4 & 73.7 & 57.1 & 85.8 & 70.7 & 83.7 & 71.0 \\
\hline $\mathrm{RF}$ & 99.4 & 92.7 & 99.3 & 90.2 & 99.7 & 75.3 & 100.0 & 84.7 & 100.0 & 80.3 & 99.7 & 84.6 \\
\hline
\end{tabular}


Table 4 Variable importance for five exotic fish species. Colored variable is the most important factor for each species.

\begin{tabular}{|c|r|r|r|r|r|}
\hline & Channel catfish & \multicolumn{1}{|c|}{ Bluegill } & Largemouth bass & Smallmouth bass & Mosquitofish \\
\hline \hline Distance & 13.5 & 20.8 & 5.1 & 12.8 & 11.0 \\
\hline Slope & 23.1 & 37.5 & 19.5 & 13.6 & 17.8 \\
\hline Depth (Spring) & 10.0 & 19.8 & 12.6 & 14.2 & 9.8 \\
\hline Depth (Autumn) & 27.5 & 18.7 & 13.1 & 12.9 & 12.4 \\
\hline Velocity (Spring) & 10.9 & 29.4 & 10.7 & 16.3 & 13.4 \\
\hline Velocity (Autumn) & 7.9 & 23.7 & 11.0 & 14.7 & 7.7 \\
\hline Tempareture (Spring) & 14.1 & 10.9 & 13.7 & 21.2 & 17.6 \\
\hline Tempareture (Autumn) & 12.8 & 17.1 & 14.7 & 21.4 & 13.4 \\
\hline
\end{tabular}

(a) Inverse of slope
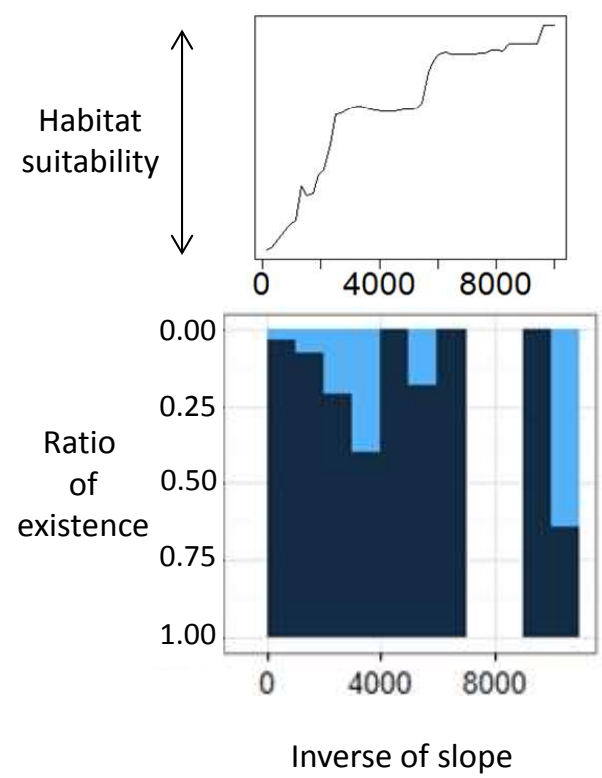

(b) Velocity at spring sampling
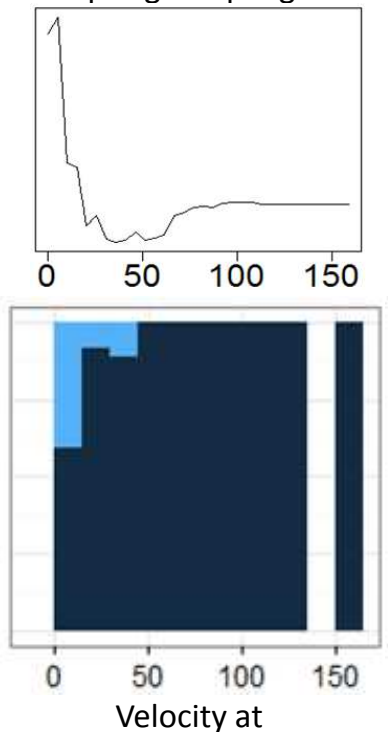

spring sampling

$(\mathrm{cm} / \mathrm{s})$ (c) Velocity at autumn sampling
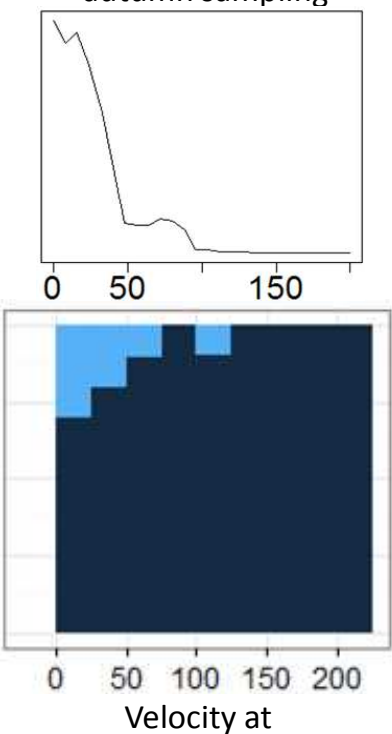

autumn sampling

$(\mathrm{cm} / \mathrm{s})$

Figure 3 Habitat suitability and its correspondence to the observation (test data). Figures in upper panels are habitat trend of bluegill. Solid lines are the magnitude of existence. Figures in lower panels are the ratios of existence of bluegill. Blue bars show the ratio of existence at each value of the explanatory variables. No colored range means that there is no sample at the range.

correspondents in red and gray colored arrows. However, there is a difference between them, which is indicated by an orange arrow. One possible interpretation of this spot is "possible habitat area" where the environmental conditions are suitable for the fish. The model projects fish's existence of the points, however, not observed by survey. This suggests that these points are preferable for targeted fish. However, they have not yet colonized or already been existed, but fail to be observed. Thus, we can devote monitoring resources into this "possible habitat area" to protect the natural condition. However, this result leaves much room for discussion. To improve this map, one possible way is special interpolation of the map. In this study, only "sample points" are showed. In order to apply practical use, it is beneficial to know whole continuous habitat information of the rivers. This could be achieved using additional information such as GIS and outputs of hydraulic model.

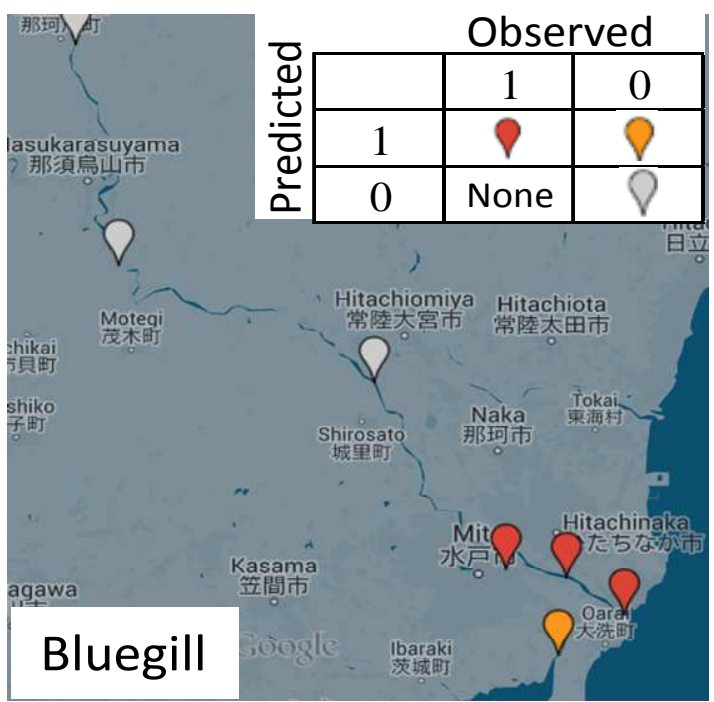

Figure 4 Projection map of Naka river 


\section{CONCLUSIONS}

SDMs of five fish species were developed using survey data both CART and RF. The result shows that the NCRE census data are appropriate for SDMs. This result also suggests that our approach have a possibility to apply for native species too. Additionally, statistical model gives some insights about their habitat information and inherit the ability for practical use. For the next step, Japan-wide analysis may be conducted with other environmental variables in order to improve the river environmental monitoring and management

ACKNOWLEDGMENT: This research was supported by JSPS-KAKENHI projects (\#25241024 (PI. Takemon Yasuhiro), \#15K00592 (PI. Yoshimura Chihiro)). We would also like to thank Dr. Masayoshi Ryo for providing us useful comment for improving this paper.

\section{REFERENCES}

1) Guisan, A. \& Thuiller, W. Predicting species distribution: Offering more than simple habitat models. Ecol. Lett. 8, 993-1009 (2005).

2) Conti, L., Comte, L., Hugueny, B. \& Grenouillet, G. Drivers of freshwater fish colonisations and extirpations under climate change. Ecography (Cop.). 38, 510-519 (2014).

3) França, S. \& Cabral, H. N. Predicting fish species richness in estuaries: Which modelling technique to use? Environ. Model. Softw. 66, 17-26 (2015).

4) Ministry of Land Infrastructure Transport and Tourism. River Enviromental database. at <http://mizukoku.nilim.go.jp/ksnkankyo/>

5) Ministry of Land Infrastructure Transport and Tourism. Fundamental servey manual. (2006).

6) Ministry of Land Infrastructure Transport and Tourism. Literature list using National Censuses on River Environments data. (2011). at <http://www.mlit.go.jp/river/shinngikai_blog/mizubekoku tyou/dai06kai/dai06kai_siryou6-1-2.pdf $\rangle$

7) Kawanabe, H., Mizuno, N. \& Nakamura, T. River Ecology. (KODANSYA, 2013).

8) Strayer, D. L. Alien species in fresh waters: Ecological effects, interactions with other stressors, and prospects for the future. Freshw. Biol. 55, 152-174 (2010).

9) Ministry of the Environment. Announcement of fourth Red List (fresh and brackish water). at <http://www.env.go.jp/press/16264.html>

10) Onishi, F. GIS Map Book for Japanese River Basin. (Osaka Municipal University Press, 2013).

11) Miyawaki, S. \& Washitani, I. Invasive alien plant species in riparian areas of Japan: the contribution of agricultural weeds, revegetation species and aquacultural species. Glob. Environ. Res. 10, 89-101 (2004).

12) Ministry of the Environment. Invasive Alien Species Act. at <http://www.env.go.jp/nature/intro/index.html>

13) Ministry of Land Infrastructure Transport and Tourism. Fundamental servey manual for fish. (2006).

14) Cutler, D. R. et al. Random Forests for Classification in Ecology. Ecology 88, 2783-2792 (2007).

15) Yu, Z., Haghighat, F., Fung, B. C. M. \& Yoshino, H. A decision tree method for building energy demand modeling. Energy Build. 42, 1637-1646 (2010).

16) De, G. \& Fabricius, K. E. Classification and Regression Trees : A Powerful Yet Simple Technique for Ecological Data Analysis. Ecology 81, 3178-3192 (2000).

17) Gelfand, S. B., Vision, C. \& Lafayette, W. AN ITERATIVE GROWING AND PRUNING ALGORITHM FOR CLASSIFICATION TREE DESIGN. in Systems, Man and Cybernetics 818-823 (1989).

18) Lee, T.-S., Chiu, C.-C., Chou, Y.-C. \& Lu, C.-J. Mining the customer credit using classification and regression tree and multivariate adaptive regression splines. Comput. Stat. Data Anal. 50, 1113-1130 (2006).

19) Breiman, L. Random Forests. Mach. Learn. 5-32 (2001).

20) Liaw, A. \& Wiener, M. Package ' randomForest '. (2015). at

<http://cran.r-project.org/web/packages/randomForest/ran domForest.pdf $>$

21) Prasad, A. M., Iverson, L. R. \& Liaw, A. Newer Classification and Regression Tree Techniques: Bagging and Random Forests for Ecological Prediction. Ecosystems 9, 181-199 (2006).

22) Askeyev, O., Askeyev, I., Askeyev, A., Monakhov, S. \& Yanybaev, N. River fish assemblages in relation to environmental factors in the eastern extremity of Europe (Tatarstan Republic, Russia). Environ. Biol. Fishes 98, 1277-1293 (2014).

23) Fielding, A. H. \& Bell, J. F. A review of methods for the assessment of prediction errors in conservation presence / absence models. Environ. Conserv. 24, 38-49 (1992).

24) Liu, C., Berry, P. M., Dawson, T. P. \& Pearson, R. G. Selecting thresholds of ocurrence in the prediction of species distributions. Ecography (Cop.). 28, 385-393 (2005).

25) Kameyama, S., Fukushima, M., Han, M. \& Kaneko, M. Spatio-temporal changes in habitat potential of endangered freshwater fish in Japan. Ecol. Inform. 2, 318-327 (2007).

26) Dietterich, T. G. Ensemble Methods in Machine Learning. in Multiple classifier systems 1-15 (2000).

27) Ministry of the Environment. List of Regulated Living Organisms under the Invasive Alien Species Act. at <http://www.env.go.jp/nature/intro/1outline/list/L-sa-05.h tml>

28) Nakao, H., Fujita, K., Kawabata, T., Nakai, K. \& Sawada, $\mathrm{H}$. Breeding ecology of bluegill, Lepomis macrochirus, an invasive alien species, in the north basin of Lake Biwa, central Japan. Japanese J. Ichthyol. 53, 55-62 (2005).

(Received September 30, 2015) 